SULLA FORMAZIONE DELLA MATERIA COLORANTE DFLL' INDACO -

E. SCHUNCH -Philosoph. Magaz. Agoslo 1855, pay. 73.

\title{
( Estratto)
}

Non ostante le numerose ricerche fatte sull'indaco dai Chimici piu distinti, non si era fin qui potuto conoscere in quale stato questa sostanza si ritrovi nelle piante dalle quali. si estrae, e su questo sogretto furono emesse opinioni differentissime. Secondo alcuni esso esiste bello e formato nelle piante da cui deriva. Per altri, e forse per il maggior numeI'o, esso vi è contenuto allo stato d'indaco bianco. Altri finalinente ammettono che esso non esiste nel vegetabile, ina si forma nei liquidi che si ottengono da questo per un processo di fermentazione. Nessuma di queste opinioni era esente da obiezioni, od abbastanza appoggiata da dati sperimentali. Le esperienze dell' $A$. sembrano spargere qualche luce su questa questione, e porterebbero a credere che nelle piante che producono l'indaco esista una specie di glucosicle, il quale decomponendosi dia origine alla materia colorante.

L'A. ha impiegato per le sue ricerche le foglie del pastello (Isatis tinctoria), che ha coltivato espressamente. Se si trattano le foglie di questa pianta con acqua bollente o con acqua fredda, si ottiene una soluzione acida, che lasciata in contatto dell aria non fornisce alcuna traccia di materia colorante, ma che fatta bollire con acido solforico od idroclorico precipita dei focchi scuri che contengono indaco. L'acetato basico di piombo produce in questa soluzione un precipitato, che racchiude la materia capace di generare l'indaco: decomponendo difatti con acido carbonico il precipitato metallico, si ha una soluzione che fatta bollire cogli acidi fornisce dell' indigotina allo stato di grande purezza. Se invece di aggiungere un acido al liquido ottenuto dalle foglie del pastello si aggiunge ad esso della soda caustica e si lascia la soluzione a sé per qualche istante, non è più possibile ricavarne per mezzo degli acidi la materia colorante. Trattando le foglie con alcole o con etere si ottengono egualmente delle soluzioni, che fatte bollire cogli acidi producono indaco. 
bietro queste esperienze preliminari l'A. si è dato ogni cura per giungere ad isolare il principio immediato che genera rindaco colla sua decomposizione. Fisso ha pero incontrato in quest'assunto i più gravi ostacoli, giacche un tal principio stbisce delle profonde alterazioni con grande facilità. Il metodo che egli preferisce per ottencre l'intento è il seguente.

Si fanno disseccare in un luogo caldo le foglie sane del pastello, e quando sono secche si scelgono solo quelle che hanno un color verde chiaro: si riducono ancora calde in polvere e si trattano con alcole freddo in un apparecchio a spostamento. Si precipita la soluzione alcolica verde con acetato di piombo e con un poco d'ammoniaca, e si lava il precipitato con alcole freddo fino che il liquido delle lavature sia divenuto di color verde chiaro. Il precipitato cosi depurato e messo in sospensione nell' acqua viene decomposto con una corrente di acido carbonico. Il liçnido grallastro che ne risulta si separa dal carbonato di piombo, quindi si sottomette all' azione dell' idrogeno solforato per eliminare qualche traccia di piombo, ed infiue si lascia evaporare all'aria o nel vuoto sull' acido solforico.

Il prodotto che così si ottiene ò una massa gialla, trasparente, glutinosa, che può aversi allo stato secco lasciandola per qualche tempo nel vuoto asciutto in istrati sottili. L'A. propone per questa sostanza il nome d'indicana. Tamto nel preparare quanto nel disseccare tale sostamza, bisogna accuratamente cvitare di riscaldarla, perchè senza tale precauzione essa si altera, sia che si trovi esposta all' aria libera, sia che si trovi rinchiusa nel vuoto. L'A. crede che in questo caso abbia luogo un' assimilazione di acqua. La soluzione di questo prodotto ha reazione acida; non si puo però escludere il caso che tale reazione dipenda da impurita; il suo sapore è debolmente amaro e nauseoso. L'indicana riscaldata in un tubo si rigonfia e fornisce un olio bruno, nel quale si forma dopo qualche tempo una materia bianca cristallina. Fatla bollire con potassa caustica sviluppa ammoniaca. La sua soluzione acquosa si colora in giallu vivace in coutatto degli alcali caustici: essa è precipitata solo dall'acetato basico di pionbo, mentre la sua soluzione alcolica precipita anche coll' acetalo di piombo ordinario. 
La proprietà pih caratteristica dell'indicana si ò quelli di fornire indigotina allorquando si tratta cogli acidi energici. Se si aggiunge acido idroclorico od acido solforico alla sua soluzione acquosa, non ba luogo alcun cangiamento sensibile; ma se il liquido si riscalda, esso diviene immediatimente azzurro; e se è sufficientemente concentrato, facendolo bollire per qualche tempo precipita in abbondanza dei fiocchi di colore azxurro volgente al porpora. La soluzione separata dai fiocchi ha un color giallo e conticne una specie particolare di zucchero. Questi fiocchi non sono formati esclusivamente d'indigotina; di fatto essi si sciolgono in gran parte nell'alcole freddo e meglio ancora nell' alcole caldo, formando una sohuzione porporina: trattando la parte che rimane indisciefta con nuovo aleole bollente, il liquido si colora in azzurro ed i fiochi acquistano un colore d'indaco puro. Se si fa evaporare ha soluzione alcolica porporina si ottiene per residno una sostanza bruna rossistra, che presenta per i suoi caratteri la più grande somighianza col rosso d' indaco di berzelius, e che l' $\mathrm{A}$. chiama indirubina . Quando si decompone l' indicana cogli acidi apparisce sempre l'indirubina assieme' all' indigotina . Quest'ultima sostanza si forma, anche a freddo, purchè si lasci agire $Y^{\prime}$ a'eido per un certo tempo, mentre l'altra prende origine solo dopo the il liquido è stato sottomesso ad una ebollizione pow lungata. Si osserva inolle che quando l' indicana è pura pro. duce una quantitì d' indigotina comparativamente maggiore di quando ha cominciato ad alterarsi. Era gia stato ripetutamente osservato il fatto, che tanto nell' indaco quanto nelke piante che lo producono si trovava sempre una materia colorante rossa accanto all' azzurra, e cío arveva indotto a supporre che fra le due sostarze vi fosse un' intimo rapporto. Le esperienze citate dimostrano, che le due materie coloran ti, almeno per il caso del pastello, sono due prodotti di decomposizione di una stessa sostanza.

E slato poco anzi arcennato che l'indicana subisce con faciliti una singolare trasformazione, in virtù della quale essa perde la proprieti di generare indaco. Tale trasformazione ha luogo ogni qualvolta l'indicana viene riscaldata, sia allo 
stato sciropposo, sia in soluzione acquosa. Nel principio l'alterazione non si manifesta con alcun segno apparente, ma proseguendo il riscaldamento l'indicana diviene quasi insolubile nell' alcole freddo, prende un color bruno e diviene precipitabile dalla sua soluzione acquosa dall'acetato neutro di piombo. Anche nel primo stadio di questa trasformazione l'indicana cessa di dare indigotina decomponendolit cogli acidi; vi è un periodo assai sfuggevole nel quale gli acidi producono indirubina, ma del resto forniscono dei fiocchi bruni, e dello zucchero come quando si adopera un prodotto inalterato. I fiocchi cosi ottenuti sono ordinariaInente formati di due materie، l'una è solubile nell'alcole, fusibile nell' acqua bollente, di aspetto resinoso, ed è stata indicata col nome d'indiretina; l'altra non si discioglie nell' alcole, è solubile invece negli alcali, somiglia molto al bruno $d$ 'indaco di Berzelius e viene chianlata indiumina.

L' alterazione dell' indicana ha luogo per l'azione del calore, come già è stato avvertito, tanto all'aria quanto nel vuoto, e pare sia effettuata istantaneamente per il solo contatto degli alcali caustici colla sua soluzione acquosa.

Lo zucchero che si forma per la decomposizione dell'indicana cogli acidi, si può avere puro precipitandolo con acetato di piombo ed anmoniaca, e decomponepdo il precipitato con idrogeno solforato. Esso non differisce per le sue proprieti dallo zucchero ottenuto dalla decomposizione degli altri glucosidi, ma sembra differirne per la composizio-

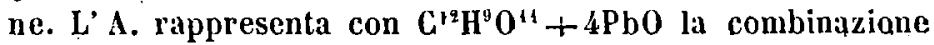
di questo zucchero coll' ossido di piombo, dalla quale deduce per lo zucchero libero la formula $\mathrm{C}^{12} \mathrm{H}^{10} \mathrm{O}^{12}$.

L'A. non ha potuto analizzare l'indicana allo stato libero: ha fatto l'analisi delle sue combinarioni coll' ossido di plombo; ma esse presentano una composizione diversa secondo il diverso modo col quale vengono preparate, e sembramo contenere un prodotto gia in parte alterato. Dalle analisi fatte l' $\Lambda$. crede però poter dedurre per l'indicana la formula $\mathrm{C}^{39} \mathrm{II}^{35} \mathrm{NO}^{36}$. In contatto degli acidi l'indicana si assimilerebbe due equivalenti d'acqua, e si decomporrebbe 
in un equivalente d'indigotina e 3 equivalenti di zucchero, secondo l'equazione seguente:

$$
\begin{aligned}
& \mathrm{C}^{59} \mathrm{II}^{53} \mathrm{NO}^{56}+2 \mathrm{HO}==\mathrm{C}^{16} \mathrm{H}^{5} \mathrm{NO}^{4}+\mathrm{C}^{56} \mathrm{H}^{50} \mathrm{O}^{36} \\
& \text { Indicana In digotina } 3 \text { eq. zucchero }
\end{aligned}
$$

Per mancanza di materia l'A. non ha potuto per ora confermare sufficientemente queste formule, nè determinare quelle degli altri prodotti derivanti dalla decomposizione dell'indicana.

Dalle esperienze riferite può dedursi. $1^{\circ}$ Che l' Isatis tinctoria non contiene indaco bello e formato, nè allo stato d'indigotina, nè allo stato d' indaco bianco. $2^{n}$ Che essa contiene però una specie di glucoside solubile nell'acqua, nell' alcole, e nell' elere capace di generare indaco quando si decompone cogli acidi. $3^{0}$ Che questo principio si allera colla più grande fucilita, sia per il contatto degli alcali canstici che per l'azione del calore, e perde allora la proprieti di produrre indaco colla sua decomposizione.

Nota SULla preparazione deL SODIO.

Fino a questi ultimi tempi si era creduto che la preparazione del sodio offrisse quasi le stesse difficolta di quelli del potassio, ma i saggi fatti da Deville per ottenerlo in gr'ande, onde impiegarlo nella fabbricazione dell alluminio, hanno mostrato il contrario. Per preparare il sodio si riscalda all una temperatura sufficientemente elevata un miscuglio intimo di carbon fossile, di carbonato di soda e di carbonato di calce. L'operazione si fa in bottiglie o cilindri di ferro, ai quali si adatta il recipiente proposto da Maresca e Donuy per il potassio. Il vapore di sodio si condensa in questo recipiente, e cola allo stato fuso in un vaso contenente olio di nalta. La ficilità colla quale il sodio può ottenersi, e può fondersi in contatto dell'aria senza che si accenda, è veramente sorprendente. Esso non ofre nel maneggiarlo nessmno degl'inconvenienti che presenta il potassio; e per quanto sia molto meno ossidabile di quello che si credeva, può impiegarsi in tutti quei casi nei quali si ha bisogno di vincere delle affinità molto energiche. 